\title{
Logic and symbolism of switchable porous
}

\section{framework materials}

L. Abylgazina ${ }^{1}$, I. Senkovska ${ }^{1}$, S. Kaskel ${ }^{1 *}$

${ }^{1}$ Inorganic Chemistry Center I, Department of Chemistry, Technische Universität Dresden, Bergstr. 66, 01069, Dresden, Germany.

*To whom correspondence should be addressed:

stefan.kaskel@tu-dresden.de 
Abstract

Metal-Organic Frameworks (MOFs) are highly porous materials composed of organic linkers connected by inorganic nodes. A unique subset of MOFs shows switchability, the ability to switch between at least two distinct structures differing significantly in porosity. These unique guest dependent pore opening and closing processes offer new opportunities in gas separation, selective recognition, sensing and energy storage. However, the factors affecting switchability are poorly understood. Network topology, micromechanics of building blocks and their hinges, but also particle size, defects, agglomeration, desolvation conditions etc. are convoluted into the responsiveness of the system.

In essence all factors are a consequence of the materials history including synthesis procedure and desolvation but also all subsequent handling steps such as mechanical and adsorption stress leading to a complex interplay of factors which are difficult to express clearly by ordinary writing systems, chemical or mathematical symbols without loss of intuitive understanding. Here we propose a symbolic language for the rationalization of switchability emphasizing the history dependent responsivity of many dynamic frameworks and their stimuli induced $1^{\text {st }}$ order phase transitions. Color representations of the guest and host offer an intuitive understanding of switchability phenomena even for non-experts. The system follows a bivalent logic inspired by Freges Begriffsschrift providing a fundamental logic structure for the rationalization of statements and representation of logic gates. 


\section{Introduction}

Metal-Organic Frameworks (MOFs) are a relatively young class of porous materials with unprecedented porosity and functionality. ${ }^{1,2}$ Commercialization is in an early stage. Several small and medium size enterprises have started to offer the porous adsorbents for various applications. Even more intriguing are the properties of these materials and their wide ranging functionality.

A unique feature of metal-organic frameworks is flexibility: A subset of MOFs can adaptively change their pore size stimulated by a guest molecule when the molecule enters the frameworks' pores. This phenomenon has been initially recognized by Li and Kaneko in a framework today termed ELM-11 and Kitagawa proposed by the term "3rd generation of MOFs". 3,4 The terms "flexible", "switchable" and "soft porous" solids for these materials are now used synonymously. A more rigorous definition is discussed in the work by Evans. ${ }^{5}$

The term "switchability" emphasizes the digital character of the transition as in many cases the huge volume change of the phase transition enforces a $1^{\text {st }}$ order transformation mechanism. Bistability of the empty host is a prerequisite for switchability and the two states resemble the simplest unit of a binary digital architecture (e.g. closed pore, $c p=0$, open pore, $o p=1$ ). Certainly this binary logic view is a stark simplification but it allows to rationalize the complex behavior as will be demonstrated below. Hence, in this work we emphasize the aspect of switchability and its connection to binary numeral systems and bivalent logic.

Since their early discovery several MOFs have been synthesized showing various types of structural transformations and even counterintuitive behavior such as negative gas adsorption (NGA) were discovered. ${ }^{6,7}$ A deeper insight into the field is summarized in recent perspectives and reviews. ${ }^{5,8,9}$ 
In the following we only explain briefly the most archetypical framework transformations in the field of switchable MOFs namely "gating" and "breathing". Gating (or gate opening) was the earliest discovery in a system now termed ELM-11. ${ }^{3}$ Gating characterizes a MOF which has little or no porosity in the guest-free phase (cp $=$ closed pore, or $n p=$ narrow pore phase) but opens the pores for a specific guest (op $=$ open pore) at a characteristic "gate pressure" of the guest molecule (or activity coefficient of the guest). As a typical example the closed and open structure of DUT-8 are displayed in figure $1 a^{10}$ This behavior results in a characteristic adsorption isotherm which is basically a combination of two isotherms characteristic for a non porous (type II) and a highly microporous type I isotherm which are connected by a steep, almost vertical line at the gate pressure characterizing the $1^{\text {st }}$ order transformation of the framework from closed to open pore. As typical for $1^{\text {st }}$ order phase transitions the transformation shows a hysteresis and closing occurs at much lower pressures close to the equilibrium transition pressure.

Breathing, instead characterizes a 2-step process (Figure 1b): The guest free MOF has its stability minimum in the open pore form (here also termed $I p=$ large pore). The filling of the pores with a guest leads to adsorption stress contracting the framework at a characteristic pressure leading to a narrow pore phase (np). At higher pressure the MOF reopens again to the guest filled Ip phase.

The underlying thermodynamics, micromechanics, and mechanisms of switchable MOF transformations have been extensively illustrated by Coudert, Evans, Maurin, Miyahara, Neimark, and van Speybroeck through simulations as discussed in detail in their reviews and will not be repeated here..$^{5,8,9,11-15}$ 

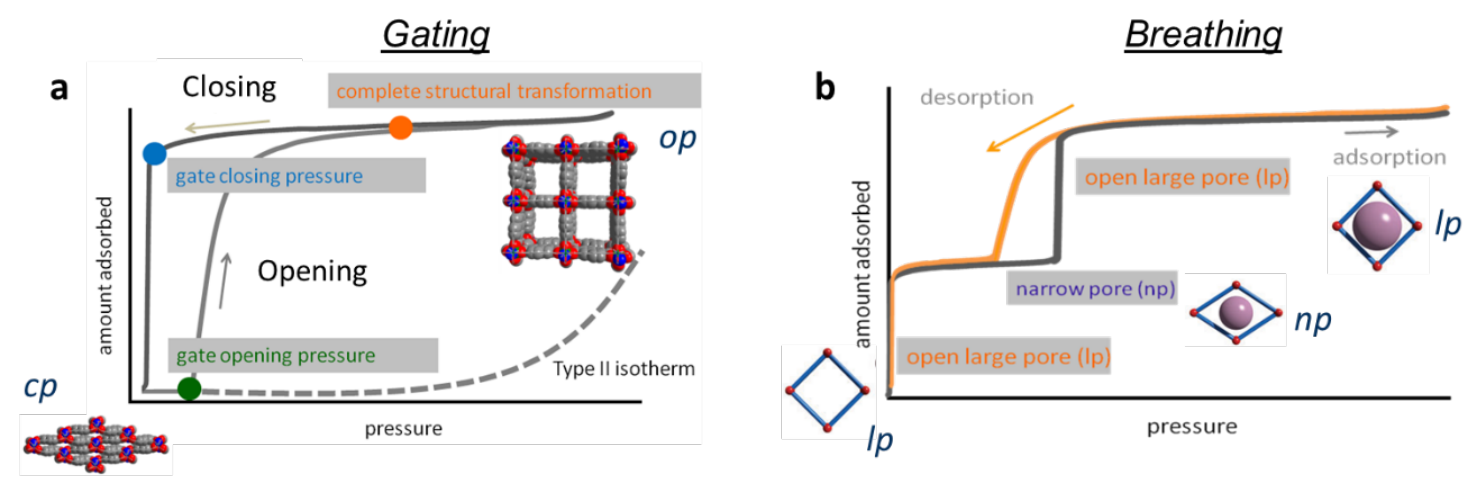

Figure 1. Adsorption isotherms for switchable MOFs. a Gating processes are characterized by a one-step opening (adsorption) and closing (desorption) step. b Breathing traverses in two steps from empty $l p$ by increasing guest pressure via partially filled $n p$ to a completely filled $I p$ phase.

However, several aspects affecting switchability are not yet included in simulations and also their exact experimental observation and description is only at the very beginning. Whether a framework is responsive to a specific stimulus or not depends not only on the crystal structure but on many determining factors. The idealized crystal structure, in particular the topology and deformability of the nodes, their hinges and the linkers defines the basis for switchable solids. However, a crystal also has a "real structure", a term encompassing finite size, domain architecture, defects etc. presumably also impacting responsivity. The most critical factors severely influencing switchability are particle size, surface deformation, domain formation, defects, etc. and these superimpose on network topology and framework composition. Not all of these factors are easily quantifiable by analytical methods. Moreover, it is reasonable to assume that a number of yet unidentified (unknown) factors affecting switchability exist.

However, these secondary factors are imprinted into the material by synthesis conditions, desolvation procedures, handling, exposure to atmosphere or defined gases, repeated switching etc. In other words: These secondary factors contain information on the history of the material. ${ }^{5}$ 
A key aspect for understanding switchability and responsivity hence lies in a visualization and documentation of the history of such dynamic porous materials. Ordinary language has limitations to document and describe intuitively the history of a material and the consequences on its properties.

This is our motivation for proposing a new logico-symbolic language describing the responsive behavior of a material and its history dependence. We will demonstrate the advantage of such a symbolic language in particular for complex history dependence of switchability.

This proposal is not dogmatic and we expect further development by other scientists in the future. We propose this language for switchable metal-organic frameworks, but it may as well be adapted to describe also other history dependent materials changes as they are of importance in technological fields such as metallurgy, ceramics, or even biomaterials.

The main postulates for our language are the following:

1. Capture unambiguously the history (trajectory in time) of a material.

2. Offer an intuitive recognition of factors and how they influence responsivity.

3. Provide a basic structure to derive logic implications, conclusions and relations between arguments.

The history of logics is closely related to the history of language development and symbols representing things. Frege, for a long time only recognized by the very few (e.g. Wittgenstein) is nowadays considered among the pioneers of modern logics. His two-dimensional language proposed in the Begriffsschrift (a formula language, modeled upon that of arithmetic, for pure thought) connecting arguments, judgements 
and signs by lines resembles in a way a logic circuit. Early readers even speculated, his logic constructions could be verified by passing electric current through them. ${ }^{16}$ On the other hand, Freges signs are abstract letters and not intuitive. Oliver Byrne in 1847 published "The First Six Books of The Elements of Euclid" with colored diagrams and symbols. ${ }^{17}$ His drawings may nowadays be recognized as artistic since the colored graphs in many ways find reminiscences in "modern" art of Theo van Doesburg and Pieter Mondriaan termed neoplasticism around 1917. However, Byrnes mission and passion was to develop an intuitive language of the very earliest mathematical work, the geometry of Euclid. His intentions were focused on educating young pupils, "to raise curiosity, and to awaken the listless and dormant power of younger minds". ${ }^{17}$

"The use of colored symbols, signs, and diagrams in the linear arts and sciences renders the process of reasoning more precise, and the attainment more expeditious". Another aspect is that the visualization makes the "retention by memory much more permanent". ${ }^{17}$

The use of symbols in chemistry has a long tradition. Early examples are symbols for elements used by alchemists such as $q$ (copper) or $\delta$ (iron). Berzelius proposed the use of letter codes for elements as it is still used today $(\mathrm{C}=$ carbonium, $\mathrm{O}=$ oxygenium, $\mathrm{Au}=$ aurum etc.) in chemical formula and structural drawings. Crystallography successfully developed symbols for symmetry operations (letters and graphs) and space group representations for a systematic differentiation of differing symmetries. This idealization is powerful for rationalization of complex crystal structures but has also limitations in particular for the rationalization of important crystal properties related to the "real structure" (e.g. conductivity, mechanical and thermal properties, reactivity) affected by particle size, morphology, grain boundaries, defects, non-stoichiometry and many more aspects. 
Materials development and rationalization today enormously profits from digitalization. The digital twin, machine learning and data mining offer tremendous opportunities also in the field of dynamic porous materials. The most extensive description and analysis of the spatiotemporal evolution of a switchable framework will be accomplishable in future only using modern in silico simulations. ${ }^{5}$

However, this quantitative analysis will be mostly accessible to the expert. Understanding theoretical analyses requires an educational background of methods and terms. A simple graphical representation of complex simulations is often a challenge. The extent of numerical data is often too massive and abstract hampering memorization. Vast collections of data may even obscure the rationalization and identification of qualitative logic interrelations.

In contrast, for communication and discussion of complex phenomena a simplified symbolic language may lead to an intuitive understanding, a more permanent retention by memory and potentially the identification of logic interrelations.

In the following we propose a logico-symbolic language for analyzing switchability inspired by Byrne and Frege. We do not claim that this proposal satisfies the rigorous theorems of logic language. However, we see this effort as a first step into a more systematic rationalization of history dependent responsivity as a basis for logic analysis

of interrelations and their communication in experimental materials chemistry to provide an intuitive understanding of complex interrelations.

\section{Results}

The key element (judgement) of Freges Begriffsschrift is shown in Fig. 2a. A horizontal line is a "content stroke" leading for example to content $A$. The thick vertical line on the left affirms the content (or judgement) to be a „fact“. The vertical line connecting $B$ to A is the "condition stroke". This symbol states a condition, leading to a logic judgement. 
"B" is a condition for "A" to be observed, or simplified "B leads to A", which is precisely termed "logic implication". This vertical line termed "condition stroke" (B is a condition for A) we will adopt for our symbolic language in which B will represent a stimulus (condition) to change the structure of the framework (Figure 2b).

In a strict logical sense of Freges Begriffsschrift figure 2a states more precisely: "(3) A is denied and B is affirmed" does not take place, but one of the other three possibilities takes place:

(1) A is affirmed and B is affirmed

(2) $A$ is affirmed and B is denied

(4) A is denied and B is denied

For a deeper discussion of Freges Begriffschrift we refer to the original literature and supporting information $\mathrm{S} 1 .^{16}$

In our new symbolic language we target the description of the pore opening or structural transition of a porous material (MOF). We align the states and structural transformations of the framework along the vertical line as it is intuitive for chemists (Figure 2b). We use the condition stroke to depict the consecutive stimuli changing the framework structure. These structural changes, the spatiotemporal evolution of the framework, are of course highly complex. ${ }^{5}$ However, for simplification and rationalization it is easier to simplify the phenomena considering only two different states of the framework. The latter is justified for many systems showing bistable characteristics in their energy landscape. 
a
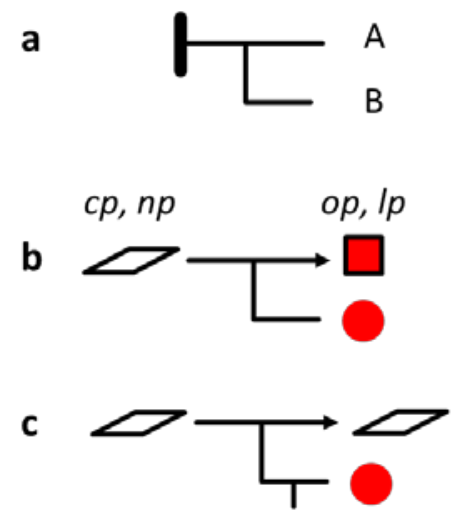

d

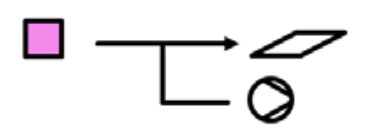

e

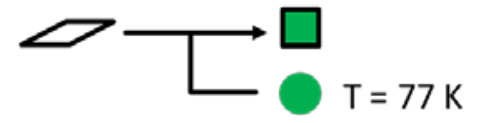

f

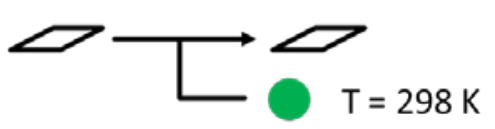

g

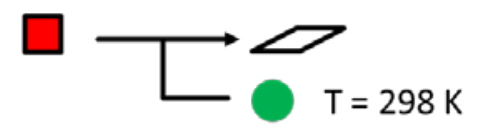

h

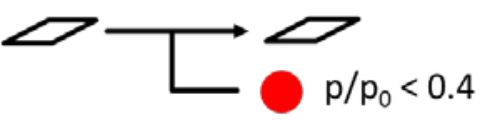

i
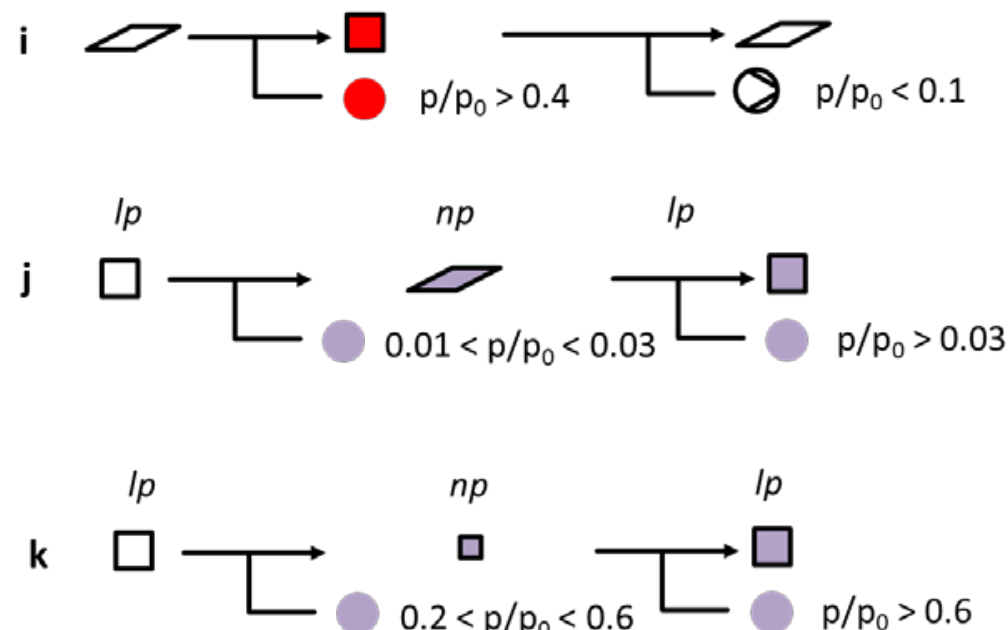

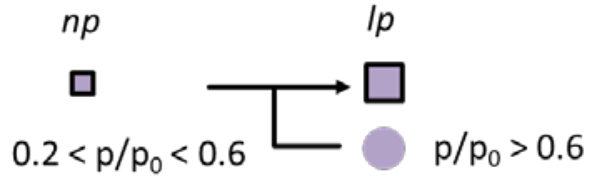

Figure 2. Elementary symbols for illustration of switching processes. a Freges original representation of a logical implication. b Elementary step representation for a gate opening process, the red circle represents the molecular stimulus (butane), the shape of the polygons represents the crystal structure of the framework. $\mathbf{c}$ The small vertical stroke represents the negation, here it represents a closed valve for the gas. $\mathbf{d}$ Desolvation, the stimulus is the symbol for a pump (evacuation). e Gate opening by nitrogen at $77 \mathrm{~K}$. $\mathbf{f}$ No gate opening by nitrogen at $298 \mathrm{~K}$. $\mathbf{g}$ Butane is desorbed by nitrogen purge at $298 \mathrm{~K}$ leading to gate closing. $\mathbf{h}$ Specification of the guest stimulus relative pressure $p / p_{0}$, if $p / p_{0}<$ gate opening pressure the framework remains closed. i The gate opening pressure is surpassed leading to gate opening in adsorption, desorption and gate closing is observed at lower relative pressure. $j$ Breathing process along the adsorption branch. $\mathbf{k}$ Alternative representation of a breathing process along the adsorption branch for a framework with cubic $c p$ phase.

At the outset for simplicity we only consider the gating process, also called gate opening, in which the MOF switches from a closed pore state $(c p)$ or narrow pore state $(n p)$ here symbolized by a squeezed rhombus into an open pore (op) or large pore (Ip) structure, symbolized by a square (Figure $2 b$ ). We omit the vertical thick "affirmation stroke" but introduce an arrow, as it is intuitive for chemists, to indicate the direction of the process in time. The arrow replaces the vertical "affirmation stroke" reflecting a transformation that is "true" in a sense that is has been observed by the experimentalists. We can line-up all structural changes along consecutive arrows as a 
history time-line for the material. The stimuli, causing structural changes ("B") are arranged below the arrow. This structure provides a clear arrangement of "transformations" and "conditions". The "condition" B responsible for the pore opening (switching) is a molecular stimulus, a gas entering the pore, for example butane, here symbolized by a red sphere. Through gate opening, the gas enters the MOF and stays inside, hence the square is filled with red color (butane) after butane exposure. A simple translation may be here "butane leads to pore opening".

This language is intuitive as the guest inclusion and structural transition is immediately understood even by a non-expert. At the same time the concept is versatile, as the condition "B" can be replaced by many symbols individually defined by the experimentalist and expanded to other stimuli. Specifications may be detailed using technical terms. The symbols for the condition "B" can be neatly arranged in tables (e.g. Table 1).

In the following we introduce the most important elementary processes for switchable MOFs with this logico-symbolistic language:

- "Butane leads to pore opening" (Figure 2b).

- "Without butane there is no pore opening" (Figure 2c).

- "Vacuum exposure leads to desolvation and pore closing" (also termed activation, Figure 2d).

The symbols are illustrated in table 1. For this publication we have entered all symbols used in the following in one table which may be confusing in the beginning. However, it should be pointed out that many experimental studies only use a limited number of gases (stimuli) and hence need to specify only a few symbols preserving the simplistic character of the symbolic language. 
Table 1: Explanation of color codes and meaning of symbols used. The specification of temperature and pressure is recommended but may be dispensable if invariable or specified elsewhere.

\begin{tabular}{|c|c|c|c|}
\hline Stimulus & Symbol & $T / \mathrm{K}$ & $p / \mathrm{Pa}$ \\
\hline$n$-Butane & & 298 & 101300 \\
\hline DMF & & 298 & $101300^{b}$ \\
\hline Nitrogen & & tbd & 101300 \\
\hline Xenon & & tbd & tbd \\
\hline $\mathrm{CO}_{2}$ & & tbd & tbd \\
\hline $\mathrm{CH}_{4}$ & & tbd & tbd \\
\hline $\operatorname{DCM}\left(\mathrm{CH}_{2} \mathrm{Cl}_{2}\right)$ & & 298 & $101300^{b}$ \\
\hline Ethanol & & 298 & tbd \\
\hline Hydrogen & & 298 & 101300 \\
\hline Oxygen & & 298 & 101300 \\
\hline Water & & 298 & $30000^{c}$ \\
\hline Vacuum & 8 & tbd & tbd \\
\hline
\end{tabular}

${ }^{a}$ tbd $=$ to be defined (specified) by the experimentalist, ${ }^{b}$ in case of liquids $p$ refers to the outer pressure, not to the vapor pressure. ${ }^{c}$ as a realistic example the vapor pressure was selected slightly below the saturation pressure.

The column for $T$ and $p$ and other important parameters can be added to specify the symbols further in detail depending on the study and variation of conditions. For many systems it may be also helpful to specify the relative pressure $p^{\prime} p_{0}$ or a pressure range instead of absolute $p$.

We further adopt Freges short vertical stroke in the conditional branch, to negate, i.e express "B" does not take place, in fact blocking the access of butane to the porous material (Figure 2c). This representation is also rather intuitive resembling a closed valve similar to a water lock.

Figure $2 d$ symbolizes what is often called "activation" in porous materials science, an inadequate wording, as it contains no information with respect to the experiment or process. "Activation" of porous solids is more appropriately termed "desolvation" and 
is characterized by guest removal from the pores (typically by vacuum or in gas flow) at defined pressure $p$ (or pressure range) and a temperature $T$ (or temperature program). For studies including varying adsorption temperatures the temperature of the guest should be specified next to the symbol (Figure 2 e,f).

With this language and expansion of table 1 important experimental observations are intuitively symbolized:

- Nitrogen at $77 \mathrm{~K}$ leads to pore opening (Figure 2e).

- Nitrogen at $298 \mathrm{~K}$ does not lead to pore opening (Figure $2 \mathrm{f}$ ).

- Purging a butane filled framework with nitrogen at $298 \mathrm{~K}$ leads to pore closing (Figure 2g).

The gate opening pressure is the characteristic pressure at which the MOF opens its pores. It is a characteristic quantity and specific for the interaction of the gas with the porous solid. We specify the guest pressure in the scheme next to the symbol (Figure $2 \mathrm{~h}, \mathrm{i})$. These statements in a logical way reflect: The MOF opens at a particular gate pressure (in figure $4 i$ at $p / p_{0}=0.4$ for adsorption switching). In this case the specific information in the table is omitted and directly transferred to the graph. An alternative graphical representation is outlined in the supporting information (section S2). This scheme also takes into account the hysteresis resulting in a different desorption switching pressure (in figure $2 i$ at $p / p_{0}=0.1$ for desorption switching).

In a more general sense the pressure is recognized as the characteristic switching pressure. This generalization also easily symbolizes breathing behavior of frameworks. The most archetypical system is MIL-53 which shows a consecutive transformation from Ip $\rightarrow \mathrm{np} \rightarrow \mathrm{Ip}$ during successive increasing the stimulus pressure (Figure 2j). Here the data approximately reflect the responsive behavior against xenon at $298 \mathrm{~K}$ as 
reported by Ferey. ${ }^{18}$ In principle the same symbols may also represent the breathing of DUT-49 leading to NGA but a crystallographer may find it more intuitive to represent this by square symbols as both forms, Ip and np are cubic (Figure $2 \mathrm{k}$ ). ${ }^{19}$ Xe has been used to analyze the transitions as the ${ }^{129} \mathrm{Xe}$ NMR chemical shift is highly sensitive to the pore size and allows to analyze NGA, gate opening and breathing easily among multiple other versatile in situ analysis techniques. ${ }^{10,20}$

\section{History dependent responsivity}

After introducing the basic symbols it is possible to represent more complex processes. In particular the complex history dependent responsivity is an important target for the communication of more complex observations. Our symbolic language is ideally suited to represent consecutive processes and transformations. An important processing step is the desolvation in vacuum before the framework is exposed to other gases as outlined above (Figure 3a).

Freges 2-dimensional graphs allow to arrange the consecutive conditions below each other resulting in neatly arranged structures (Figure 3b). This concepts gives also access to more complex consecutive procedures.

Figure $3 e$ describes a switchable MOF that is activated from DMF and repeatedly exposed to butane and evacuated again, and then does not open any more. This phenomenon is a characteristic aging by repeated switching reflecting a more complex sample history. ${ }^{21}$ The equivalent 2-dimensional graph is depicted in the supporting information (Section S3). 
a

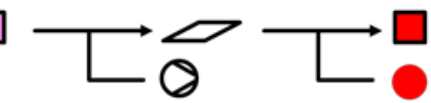

b

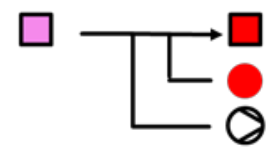

c

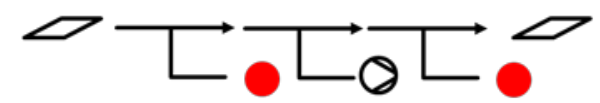

h
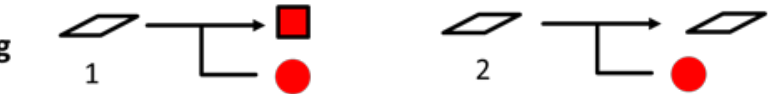

d

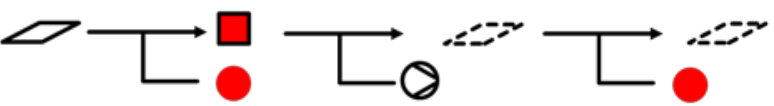

e

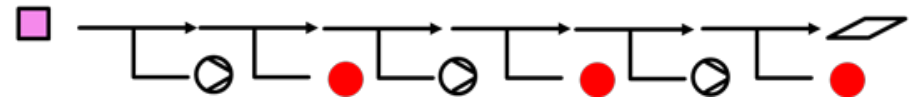

Figure 3. Representation of history dependent responsivity. a Desolvation induced pore closing followed by butane induced pore opening. b Simplified representation for desolvation induced pore closing followed by butane induced pore opening. c Representation of two consecutive butane adsorption cycles with aging (unkwnown intermediates). d Proper representation of intermediates altered through aging by adsorption cycling. e Representation of multiple consecutive butane adsorption cycles with aging (unkwnown intermediates). f Proper distinction of intermediates altered by aging using numbers. $\mathbf{g}$,h Distinction of intermediates by their responsivity.

This example (Figure 3e) shows the strength of the logico-symbolic language:

We can represent all transformation steps without explicit specification (knowledge) of intermediates. In this graph the intermediates are not symbolized because it was impossible to analyze all structural aspects in between the cycles. ${ }^{21}$ Such a situation is frequently met, if the experimentalist has not enough quantifiable data to characterize all intermediates in detail. Not knowing the cause of the changes in responsivity, inspiration and imagination is needed to guess the techniques suitable to identify the cause.

Moreover, the intrinsic logic may be used to derive simple conclusions on intermediates.

For this reason we simplify the history dependence to only two adsorption cycles (Figure 3c). 
From our earlier description we know the MOF to open with butane, and close again when it is desorbed (Figure 3f). This leaves us with two statements contradicting each other (Figure $3 g$ ), both statements can only be true if $c p(1) \neq c p(2)$ (Figure $3 h$ ). The $c p$ framework (2) after surpassing one opening and closing cycle is not identical to the starting $c p$ structure (1). In other words the material records the history (events, transformations) hidden in the "real structure" (The term "real structure" encompasses domain structure, defects, finite size etc. in contrast to the idealized defect-free periodic crystal structure). After recognizing these changes, the observer could then indicate the changes in the "real structure" symbolically even if one does not know exactly the origin or descriptor characterizing this change (Figure 3d). For simplicity we have introduced here arbitrarily a broken line. The broken line could indicate some degree of structural "deterioration" implying defect formation, milling, or surface deformation. For DUT-8(Ni) the huge volume change indeed causes cracking (shattering) of larger crystallites leading to a domain structure. ${ }^{21}$ The domain boundaries generate additional strain in the crystals changing their adaptive behavior causing framework stiffening. Hence, in this case the broken line is (more or less) intuitive. In principle other symbolizations such as filling with a checked pattern would also be intuitive symbols for domain formation. However, we recommend to reserve the interior of the polygon for the guest specification while the lines represent the framework.

Framework composition or synthesis conditions dependent responsivity

We can also easily use this language to compare MOFs differing in composition. For example MOFs often differ with respect to the metal in the nodes of the framework. The following diagram shows a comparison of DUT-8(Ni) and DUT-8(Co), pillared layer 
MOFs containing $\mathrm{Ni}_{2}$ - or $\mathrm{Co}_{2}$-paddle-wheel nodes, demonstrating only the Ni-MOF to open when exposed to butane while the Co-MOF remains closed (Figure $4 \mathrm{a}, \mathrm{b}) .{ }^{22}$

Frequently, the synthesis conditions define whether a framework is switchable or not but we cannot immediately identify the cause for suppressed switchability. The frameworks differ in structure in a wider sense, but the descriptor characterizing the difference is unknown. These differences may relate to defects, small variations in chemical composition, surface termination, crystal morphology or a certain grain boundary structure induced by the particular synthesis conditions, but these differences are not immediately recognizable by the experimentalist due to methodological limitations. In this case it is important to represent the differences in synthesis conditions as part of the history of the MOF and symbolize differences in the framework by a different line color or structure (Figure 4 e,f).

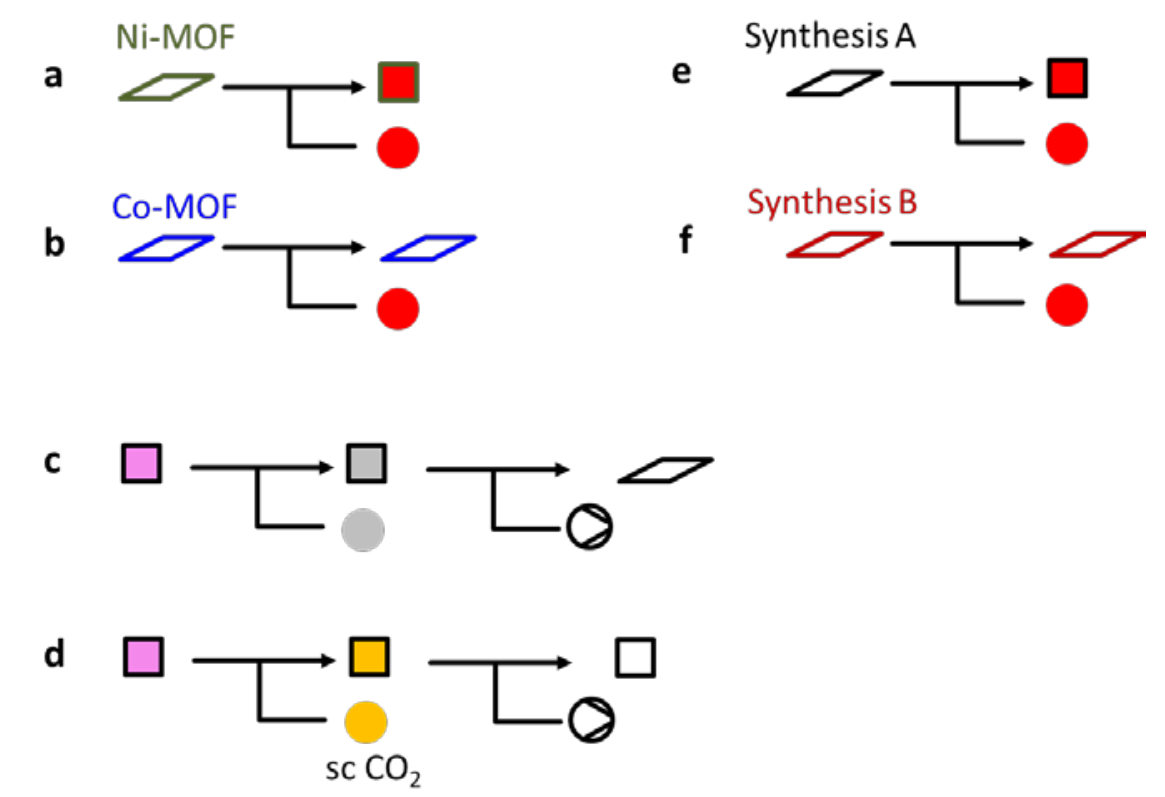

Figure 4. Dependence of responsivity related to framework structure in a wider sense (real structure). a,b Framework composition, impact of the connecting node. c,d Impact of the desolvation process. e,f Impact of synthesis conditions, unknown descriptor characterizing structural differences. 


\section{Framework desolvation dependent responsivity}

Another decisive process affecting switchability is the framework desolvation. The symbols for this process have been already introduced above.

Recently we reported the impact of framework desolvation on switchability for the model system DUT-8(Zn) and found a highly complex interdependence of particle size and activation conditions. ${ }^{23}$ For simplification here we do not consider the particle size effects and only address the responsivity of the bulk material. We compare two different desolvation conditions namely solvent exchange with dichloromethane (DCM) followed by evacuation and supercritical exchange with $\mathrm{CO}_{2}$ and simplify the observations (Figure 4c,d).

The symbolic language intuitively transfers the information that the solvent exchange leads to pore closing after DCM desolvation by vacuum, while the supercritical drying retains the op form after evacuation.

\section{Multiple phase formation}

The foregoing example also reveals a problem of our bivalent logic language. This scheme cannot easily account for the experimental observation of phase mixtures. In the real experiment here it was observed that certain desolvation procedures (in this case thermal desolvation of DMF) lead to a phase mixture of op and $c p$ phase..$^{23}$ In principle one could symbolize the formation of mixtures as demonstrated in figure 5a. However, such a language would imply the loss of all logic implications as "yes" and "no" (op and $c p$ ) are true at the same time. Instead, from a logical point of view the observation of multiple phases implies either the sample to consist of more than one "real structure" (Figure 5b), or the desolvation conditions (stimulus) are not identical for all crystals (Figure 5c). This aspect is corroborated by thermodynamic considerations as the coexistence of op and $c p$ phase in equilibrium is only allowed at a single defined 
pressure of the guest, while our switching observations (start and end points) are mostly far from equilibrium. Hence, at least one kind of crystals is not in equilibrium and at least two different species must exist.

a

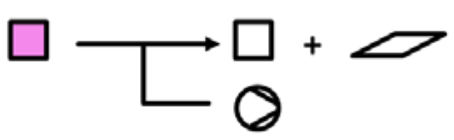

b
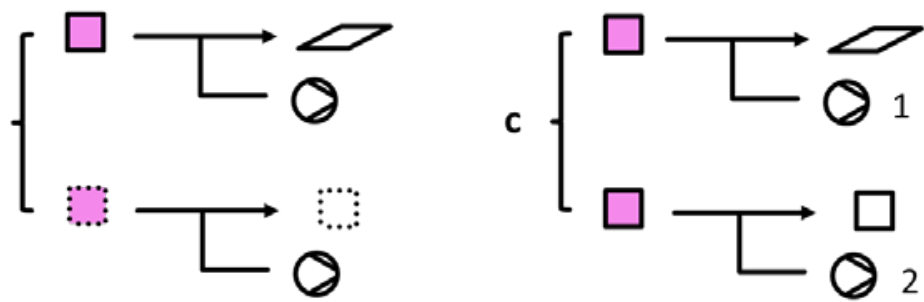

Figure 5. The problem of multiple phase formation. a Improper representation. b Proper representation of multiple phase formation indicating differences in framework structure of the starting material in a wider sense using line coding. c Representation of multiple phase formation indicating inhomogeneous desolvation conditions using number codes.

The term "real structure" difference is deliberately chosen here to cover a wider meaning and could refer to differences in particle size, defect concentration, or surface deformation, etc. A frequently assumed reason for multiple phase observation in such an example is a wider particle size distribution and for the fraction of small particles the contraction is suppressed. The implications are important as logics imply that the nonclosing particles are different in origin and the starting sample contains at least two different entities, differing in size or shape or other structural characteristics (Figure $5 b)$.

Inhomogeneous desolvation conditions (Figure 5c) on the other hand are a practical obstacle often overlooked. In the catalysis community a "shallow bed activation" is known to be crucial for good performance. If a porous system is activated as a powder, 
multiple gradients in the sample may be generated. The vapor desolvated from crystals at the bottom of a flask passes through all crystals on the top layer leading to differences in their history. Activation ports of adsorption equipment are primitive and their heating wires cause hot-spots. In such systems the desolvation temperature is not well defined. Evacuation pressures are frequently not even recorded by the observer.

An alternative explanation for multiple phase formation far from equilibrium is that some crystals disintegrate into smaller crystals during activation due to desorption stress. However, from a logical point this explanation is equivalent to the situation depicted in figure $5 b$ as these crystals disintegrating and remaining rigid somehow differ in "real structure" (size, shape, defect concentration or else) in the starting particle ensemble. In essence from a puristic logical point of view the framework symbol introduced here can only represent one individual crystal or an ensemble of "identical" crystals.

\section{Crystal size and morphology}

Recently it has been pointed out that crystal size and also morphology play a key role affecting switchability. ${ }^{24-26}$ Crystal size affects thermodynamics and kinetics of phase transitions. However, for switchable MOFs particle size effects already play a role at submicron level whereas normal binary phases display pronounced size effects only well below $100 \mathrm{~nm}$. The most prominent effect is the suppression of flexibility below a critical crystal dimension. In other words small crystals do not show switchability. We propose two alternative schemes for representation (Fig 6a, b). 


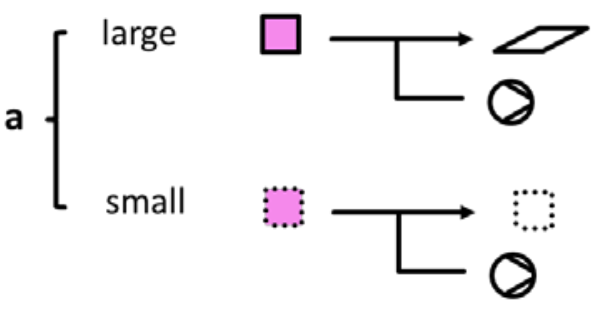

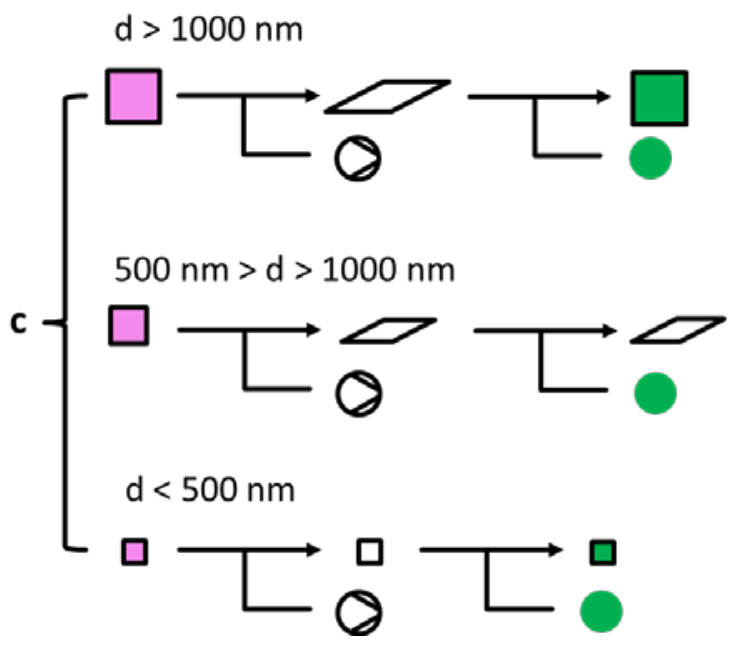

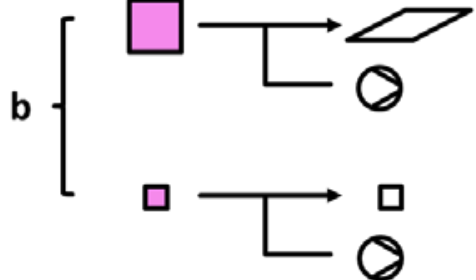

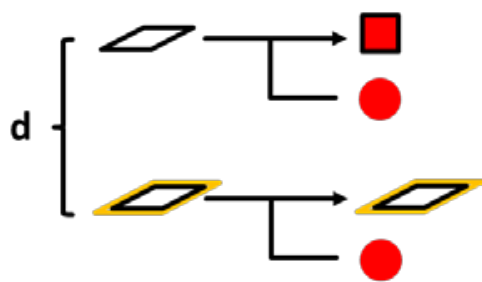

Figure 6. Effects of particle size and matrix embedding on responsivity. a Identification of finite size by line coding. $\mathbf{b}$ Representation of crystal sizes by differently sized framework symbols. c Representation of three different crystal size regimes by size-varying symbols. d Representation of altered (suppressed) responsivity by embedding in matrices or polymer adsorption on the outer surface.

Scheme $b$ also allows to easily describe more complex phenomena. For example for DUT-8(Ni) it has been shown that macro crystals are flexible and reopen with $\mathrm{N}_{2}$, while nanocrystals (<500 nm) are rigid and do not close upon desolvation. ${ }^{27}$ However, a peculiar phenomenon was observed that intermediate size crystals close but do not reopen (Figure 6c). The exact reason for such phenomena is still a matter of investigation but similar behavior has been also reported for DUT-8(Zn). ${ }^{23}$ A description of such phenomena in ordinary language requires nested if-clauses that create an aversion in the mind of the reader and are difficult to digest and memorize. Our symbolism instead allows a simple visualization and immediate perception of such more complex phenomena. 


\section{Matrix rigidification}

Crystals are never isolated entities. On earth, they are always connected to a surface, substrate, agglomerated with other crystals, intergrown, covered by side products or polymer binders used to shape MOFs into granules.

Any matrix or interfacial contact alters the responsivity. A simple observation is the matrix rigidification, a stiffening by surrounding components that suppress the pore opening process. ${ }^{28,29}$ An intuitive representation is to introduce such coating on the crystal as an additional line (Figure $6 \mathrm{~d}$ ).

\section{Selective adsorption}

A highly important application of adsorbents is adsorptive separation. Switchable MOFs frequently show guest dependent responsivity. A good example to symbolize the high selectivity observed for DUT-8(Co) (Figure 7c) in contrast to DUT-8(Ni) (Figure 7a) is the responsivity towards butane and DCM. ${ }^{22}$ While the Ni-framework shows a non-selective gate opening for both gases, the Co-framework selectively opens its pores only for DCM. 

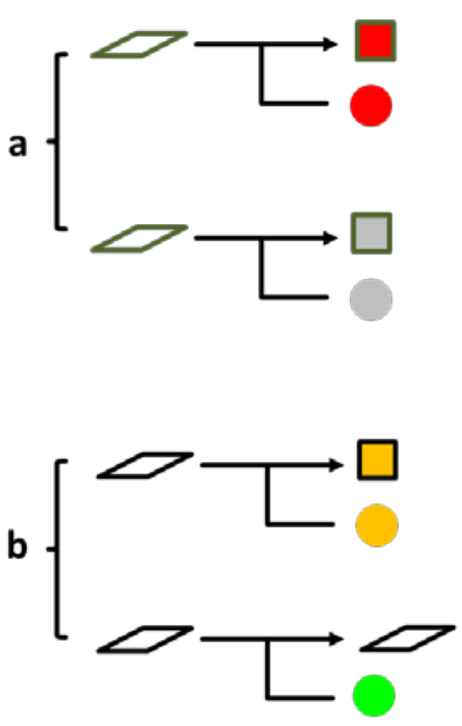

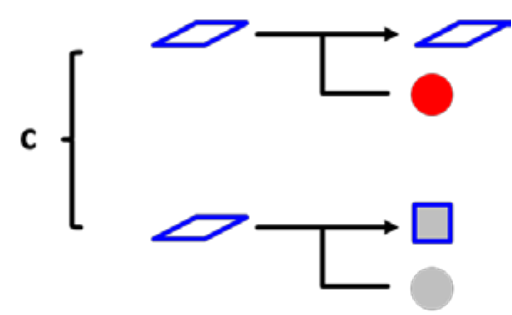

d

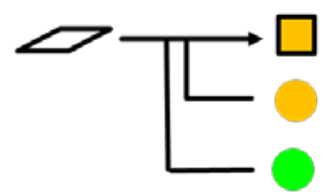

e

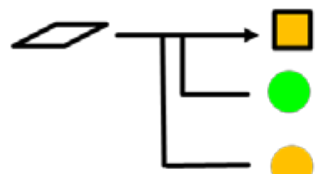

Figure 7. Selective responsivity of switchable frameworks. a Non-selective pore opening by butane and DCM (Ni-MOF). b Selective pore opening by $\mathrm{CO}_{2}$. c Selective pore opening by DCM (Co-MOF). d Selective adsorption of $\mathrm{CO}_{2}$ in gas mixture adsorption ( $1^{\text {st }}$ stimulus: $\mathrm{CH}_{4}, 2^{\text {nd }}$ stimulus $\mathrm{CO}_{2}$ ). e Selective adsorption of $\mathrm{CO}_{2}$ in mixture phase adsorption ( $1^{\text {st }}$ stimulus: $\mathrm{CO}_{2}, 2^{\text {nd }}$ stimulus $\mathrm{CH}_{4}$ ).

Another important example also considered frequently for applications is $\mathrm{CO}_{2} / \mathrm{CH}_{4}$ separation. $\mathrm{CO}_{2}$ can open certain frameworks at defined $p, T$ variable while $\mathrm{CH}_{4}$ under the same conditions cannot open the framework (Fig. 7b). The underlying reason is the adsorption enthalpy which is higher in magnitude for $\mathrm{CO}_{2}$.

The consequence is that even in mixture phase adsorption an ultrahigh selectivity for $\mathrm{CO}_{2}$ is observed as symbolized in Figure $7 \mathrm{~d}$ and e. No matter in which sequence the gases are introduced only $\mathrm{CO}_{2}$ enters the pores leading to pore opening. ${ }^{30}$ The amount of methane in the adsorbed phase is negligible. Certainly this is a stark simplification but it facilitates the rationalization. For a more in depth discussion it would be important to explicitly denote the partial pressures of the guest in the mixture gas phase which plays the role of the absolute pressure for the single phase adsorption assuming ideal solutions. 


\section{Illustration of complex dependencies}

In our view the symbolic language is in particular useful for the analysis of more complex sample histories. We only give one typical example, a guest dependent shape memory effect. In words: A switchable MOF closes the pores if it is desolvated from DCM, however, after adsorption of ethanol the desorption of ethanol does not lead to pore closing. Such a complex behavior is difficult to conceive as a sentence, but easily depicted in the symbolic language (Figure 8a).

\section{Discussion: Logic syllogism, laws of thought, logic gates}

Freges Begriffsschrift was a landmark of modern logics for the expression of judgments of pure thought. Our definitions are probably not rigorous enough to satisfy such high standards. We illustrate the chances and limitations only briefly by looking at one of the simple laws of logic, a chain syllogism.

The modern representation of a chain syllogism by higher mathematics is the following:

$$
((A \Rightarrow B) \wedge(B \Rightarrow C)) \Rightarrow(A \Rightarrow C) \quad \text { Eq. } 1
$$

Freges original two-dimensional representation is shown in figure 8b. The problem and difference to our language is that statements A, B, C in Freges logic are not classified, they operate without privilege in a column on the right hand, a distinction of subject and predicate does not occur. In our consecutive language the history aspect is emphasized by the arrow, connecting the states of the framework and their representations lined up horizontally. The "condition stroke" was up to this point reserved for the stimulus only. This classification (state vs. stimulus) is an important 
difference to Freges formula language but more intuitive for the chemist as it resembles an equation for a chemical reaction as outlined in the beginning.

For a chain syllogism we need to state a conditional relationship between two stimuli $(A \Rightarrow B)$. Figure $8 c$ illustrates a realistic example. We state the connection between two stimuli as "hydrogen and oxygen form water vapor at $298 \mathrm{~K}$ " $(A \Rightarrow B)$. We select a responsive framework opening the pores when exposed to water vapor $(B \Rightarrow C)$. The logic consequence is that this MOF will also be responsive to a mixture of hydrogen and oxygen and should open the pores $(A \Rightarrow C)$.

a
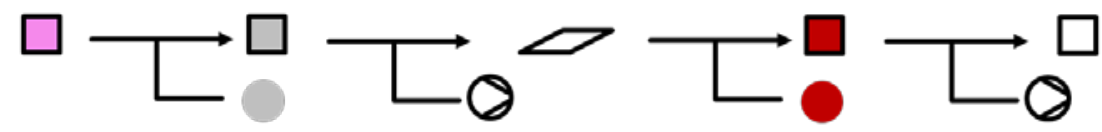

b

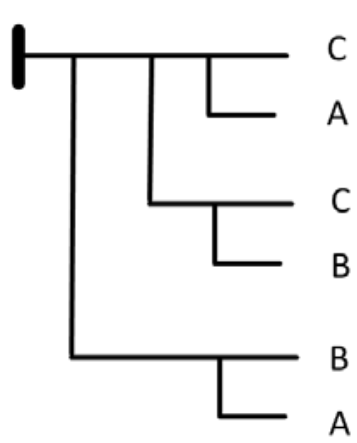

C

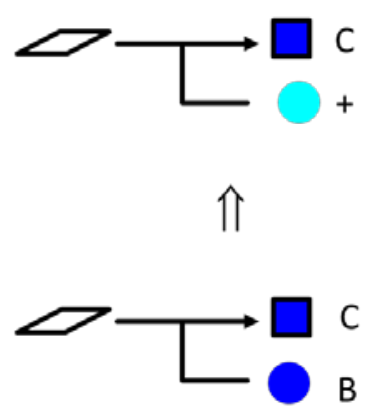

d

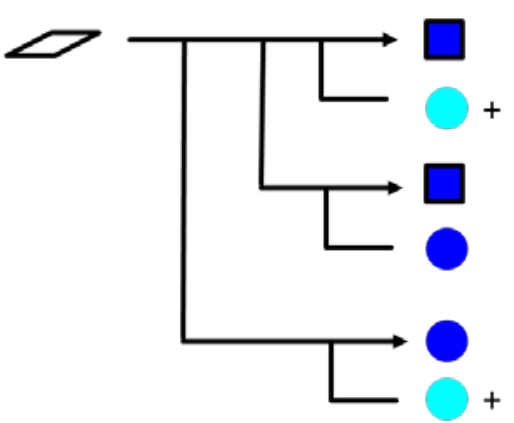

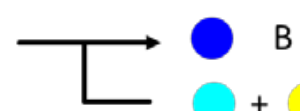

A

Figure 8. Complex interdependence and logic judgments. a Representation of solvation induced shape memory effect, resolvation of the framework with ethanol leads to a change in responsivity. b Freges original representation of a chain syllogism. c Example for chain syllogism through switchable MOFs by interlinked stimuli. d Mapping of Freges original chain syllogism with symbolic language and connected stimuli.

Several MOFs with responsivity towards water have been reported. A more recent example of an eightfold interpenetrated MOF with distinct water responsivity has been reported by Roztocki et al. ${ }^{31}$ 
This example shows an adequate transfer of Freges original scheme. However, some adaption is required in order to represent $A$ as a condition for $B$, in this example the stimuli are conditionally interrelated $(A \Rightarrow B)$. However, in principle it should be also possible to consider other general hypotheses (e.g. "all framework nanoparticles are rigid") related to the framework structure as a condition and expand the logic analyses further to more complex laws of thought elaborated in the Begriffsschrift. ${ }^{16}$ This endeavor certainly requires further development in future to derive logical implications and is beyond the scope of this work.

An appealing aspect outlined in the introduction is the identification of framework states with a binary coding $(c p=0, o p=1)$. This aspect enables the conceptual construction of logic gates. The example outlined in figure $8 \mathrm{c}-\mathrm{d}$ may serve as an illustration to realize an AND gate. It is reasonable to assume that a switchable gating system will neither open the pore by exposure to hydrogen nor to oxygen at $298 \mathrm{~K}$ (Figure 9a, b). The underlying reason is the high temperature far from the respective boiling points. On the other hand, assuming hydrogen and oxygen form water at $298 \mathrm{~K}$, the resulting water vapor will open the pore system (Figure $9 \mathrm{c}){ }^{31}$

a

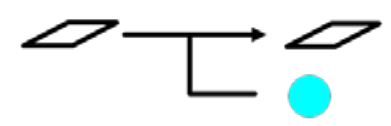

d

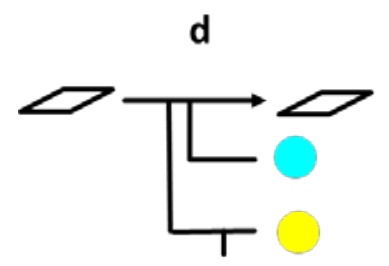

b

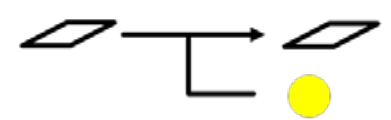

e

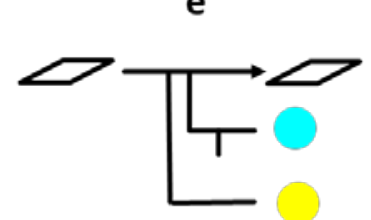

C

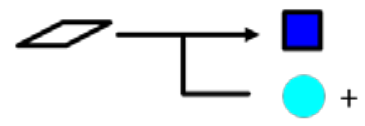

f

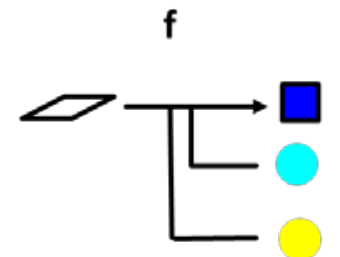

Figure 9. Logic gate realization by switchable framework. a MOF does not open with oxygen. b MOF does not open with hydrogen. c MOF opens only if oxygen and hydrogen are both present, because they form water. d MOF does not open with oxygen. e MOF does not open with hydrogen. $f$ MOF opens only if oxygen and hydrogen are both present, because they form water. 
Hence, only if hydrogen and oxygen are both present as stimuli the MOF will switch from $0(c p)$ to $1(o p)$ (Figure 9c). This is exactly the definition of a logic "AND" gate in which two statements need to be true in order to execute the logic operation. An alternative representation making use of the "negation stroke" is also illustrated in figure $9 \mathrm{~d}$-f. This example illustrates the potential of using switchable MOFs for performing logic operations. Realizing complex computing architectures with switchable MOFs is certainly beyond the scope in near future. But sensing and the simultaneous recognition of multiple stimuli may be within reach using highly selective switchable MOFs and their logic architectures. In particular the multitude of functionalized linkers and subtle hydrogen bonding interactions in multivariate MOFs provide a versatile platform for complex recognition patterns. In a sense, the very simplistic example given in figure 9 resembles an elementary logic recognition process, namely the fact that two stimuli are present at the same time leading to a qualitative structure change, a materialized memory of changes in the environment.

\section{Summary}

We have introduced a symbolic language for the visualization of switchability in porous MOFs. This symbolic language is intuitive and simple and improves the rationalization and representation of complex observations and interdependent responsivity phenomena. The guest inducing the pore opening as the stimulus is represented by a color and induced as a condition taking advantage of Freges "condition stroke" for logic representation. The states of the framework are symbolized by characteristic polygons (square and rhombus) resembling distinct open and closed (or contracted) crystalline phases in a rather simplified manner. Such a bivalent logic is certainly a stark simplification of responsivity in real materials. However, for many switchable frameworks showing bistability such representation is adequate and useful for 
rationalization and communication. The stimulus induces a first order transition. In principle this scheme can be also expanded for multiple consecutive $1^{\text {st }}$ order phase transitions but is less appropriate for continuous $2^{\text {nd }}$ order phase transitions.

The consecutive representation of multiple stimuli resolves misapprehension in the discussion of complex history dependent switchability phenomena. Color coding provides intuitive reception for a multitude of different guests, or other stimuli with defined thermodynamic conditions customized by individual authors for their studies. The importance of desolvation and crystal size is easily symbolized enabling a highly intuitive understanding even for scholars without advanced qualification or specialists in other disciplines.

An advantage of the symbolistic representation is that multiple consecutive stimuli and their impact can be visualized without detailed knowledge of the intermediates and their characteristic descriptors. The system is suited to visualize elementary logic interrelations and rationalize interdependent stimuli responses. The transfer of Freges more complex syllogism schemes is possible but requires the identification of additional conditional relations between stimuli or framework states. This is the disadvantage of categorizing statements into two classes, namely framework states vs. stimuli (conditions). Despite this shortcoming such an approach is more intuitive and the advantages of immediate perception even by non-experts largely outweigh additional efforts for direct mapping onto Freges patterns.

Hopefully, the logico-symbolistic approach will be expanded to communicate new discoveries and improve the understanding of sample history in dynamic materials changing their structure in an adaptive manner as a response to a stimulus. Exploring their logic interrelations and arranging them into higher order structures may in principle also be used to create more complex recognition patterns for sensing, to design new air logic control systems and explore complex logical architectures in future. 


\section{Acknowledgement}

Financial support by the DFG (FOR2433) is gratefully acknowledged. 


\section{References}

1. Kaskel, S. The Chemistry of Metal-Organic Frameworks (Wiley-VCH, 2016).

2. Yaghi, O. M., Kalmutzki, M. J. \& Diercks, C. S. Introduction to Reticular Chemistry: MetalOrganic Frameworks and Covalent Organic Frameworks (Wiley-VCH, 2019).

3. Li, D. \& Kaneko, K. Hydrogen bond-regulated microporous nature of copper complexassembled microcrystals. Chem. Phys. Lett. 335, 50-56; 10.1016/S0009-2614(00)01419-6 (2001).

4. Horike, S., Shimomura, S. \& Kitagawa, S. Soft porous crystals. Nat. Chem. 1, 695-704; 10.1038/nchem.444 (2009).

5. Evans, J. D., Bon, V., Senkovska, I., Lee, H.-C. \& Kaskel, S. Four-dimensional metal-organic frameworks. Nat. Commun. 11, 2690; 10.1038/s41467-020-16527-8 (2020).

6. Krause, S. et al. A pressure-amplifying framework material with negative gas adsorption transitions. Nature 532, 348-352; 10.1038/nature17430 (2016).

7. Evans, J. D., Bocquet, L. \& Coudert, F.-X. Origins of Negative Gas Adsorption. Chem 1, 873886; 10.1016/j.chempr.2016.11.004 (2016).

8. Schneemann, A. et al. Flexible metal-organic frameworks. Chem. Soc. Rev. 43, 6062-6096; 10.1039/C4CS00101J (2014).

9. Coudert, F.-X., Boutin, A., Jeffroy, M., Mellot-Draznieks, C. \& Fuchs, A. H. Thermodynamic Methods and Models to Study Flexible Metal-Organic Frameworks. ChemPhysChem 12, 247-258; 10.1002/cphc.201000590 (2011).

10. Klein, N. et al. Structural flexibility and intrinsic dynamics in the $M 2(2,6-n d c) 2$ (dabco) $(M=$ $\mathrm{Ni}, \mathrm{Cu}, \mathrm{Co}, \mathrm{Zn})$ metal-organic frameworks. J. Mater. Chem. 22, 10303-10312; 10.1039/C2JM15601F (2012).

11. Sarkisov, L., Martin, R. L., Haranczyk, M. \& Smit, B. On the Flexibility of Metal-Organic Frameworks. J. Am. Chem. Soc. 136, 2228-2231; 10.1021/ja411673b (2014).

12. Vanduyfhuys, L. et al. Thermodynamic insight into stimuli-responsive behaviour of soft porous crystals. Nat. Commun. 9, 204; 10.1038/s41467-017-02666-y (2018).

13. Evans, J. D., Krause, S., Kaskel, S., Sweatman, M. B. \& Sarkisov, L. Exploring the thermodynamic criteria for responsive adsorption processes. Chem. Sci. 10, 5011-5017; 10.1039/C9SC01299K (2019).

14. Neimark, A. V., Coudert, F.-X., Boutin, A. \& Fuchs, A. H. Stress-Based Model for the Breathing of Metal-Organic Frameworks. J. Phys. Chem. Lett. 1, 445-449; 10.1021/jz9003087 (2010).

15. Numaguchi, R., Tanaka, H., Watanabe, S. \& Miyahara, M. T. Simulation study for adsorptioninduced structural transition in stacked-layer porous coordination polymers: Equilibrium and hysteretic adsorption behaviors. J. Chem. Phys. 138, 54708; 10.1063/1.4789810 (2013).

16. van Heijenoort, J. From Frege to Gödel (Harvard University Press, 1967).

17. Oechslin, W. \& Lamers-Schutze, P. Six Books of Euclid (Taschen America, LLC, 2010).

18. Boutin, A. et al. Breathing Transitions in MIL-53(Al) Metal-Organic Framework Upon Xenon Adsorption. Angew. Chem., Int. Ed. Engl. 48, 8314-8317; 10.1002/anie.200903153 (2009).

19. Kolbe, F. et al. High-Pressure in Situ 129Xe NMR Spectroscopy: Insights into Switching Mechanisms of Flexible Metal-Organic Frameworks Isoreticular to DUT-49. Chem. Mater. 31, 6193-6201; 10.1021/acs.chemmater.9b02003 (2019).

20. Bon, V., Brunner, E., Pöppl, A. \& Kaskel, S. Unraveling Structure and Dynamics in Porous Frameworks via Advanced In Situ Characterization Techniques. Adv. Funct. Mater. 30, 1907847; 10.1002/adfm.201907847 (2020).

21. Bon, V., Kavoosi, N., Senkovska, I. \& Kaskel, S. Tolerance of Flexible MOFs toward Repeated Adsorption Stress. ACS Appl. Mater. Interfaces 7, 22292-22300; 10.1021/acsami.5b05456 (2015).

22. Ehrling, S. et al. Crystal size versus paddle wheel deformability: selective gated adsorption transitions of the switchable metal-organic frameworks DUT-8(Co) and DUT-8(Ni). J. Mater. Chem. A 7, 21459-21475; 10.1039/C9TA06781G (2019). 
23. Abylgazina, L. et al. Tailoring adsorption induced switchability of a pillared layer MOF by crystal size engineering. CrystEngComm; 10.1039/D0CE01497D (2021).

24. Sakata, Y. et al. Shape-Memory Nanopores Induced in Coordination Frameworks by Crystal Downsizing. Science 339, 193; 10.1126/science.1231451 (2013).

25. Krause, S. et al. The effect of crystallite size on pressure amplification in switchable porous solids. Nat. Commun. 9, 1573; 10.1038/s41467-018-03979-2 (2018).

26. Kundu, T., Wahiduzzaman, M., Shah, B. B., Maurin, G. \& Zhao, D. Solvent-Induced Control over Breathing Behavior in Flexible Metal-Organic Frameworks for Natural-Gas Delivery. Angew. Chem., Int. Ed. Engl. 58, 8073-8077; 10.1002/anie.201902738 (2019).

27. Miura, H. et al. Tuning the gate-opening pressure and particle size distribution of the switchable metal-organic framework DUT-8(Ni) by controlled nucleation in a micromixer. Dalton Trans. 46, 14002-14011; 10.1039/C7DT02809A (2017).

28. Hou, J. et al. Metal-organic framework crystal-glass composites. Nat. Commun. 10, 2580; 10.1038/s41467-019-10470-z (2019).

29. Kriesten, M. et al. Shaping of Flexible Metal-Organic Frameworks: Combining Macroscopic Stability and Framework Flexibility. Eur. J. Inorg. Chem. 2019, 4700-4709; 10.1002/ejic.201901100 (2019).

30. Sin, M. et al. In Situ 13C NMR Spectroscopy Study of CO2/CH4 Mixture Adsorption by Metal-Organic Frameworks: Does Flexibility Influence Selectivity? Langmuir 35, 3162-3170; 10.1021/acs.langmuir.8b03554 (2019).

31. Roztocki, K. et al. Collective Breathing in an Eightfold Interpenetrated Metal-Organic Framework: From Mechanistic Understanding towards Threshold Sensing Architectures. Angew. Chem., Int. Ed. Engl. 59, 4491-4497; 10.1002/anie.201914198 (2020). 